OPEN ACCESS

Edited by:

Arianna Morozzi,

Istituto Nazionale di Fisica Nucleare di

Perugia, Italy

Reviewed by:

Paola Ferrario,

Donostia International Physics Center

(DIPC), Spain

Carlo Fiorini,

Politecnico di Milano, Italy

${ }^{*}$ Correspondence:

Craig S. Levin

cslevin@stanford.edu

Specialty section:

This article was submitted to Radiation Detectors and Imaging, a section of the journal

Frontiers in Physics

Received: 16 November 2021 Accepted: 05 January 2022 Published: 26 January 2022

Citation:

Gonzalez-Montoro A, Pourashraf S,

Cates JW and Levin CS (2022)

Cherenkov Radiation-Based

Coincidence Time Resolution

Measurements in BGO Scintillators.

Front. Phys. 10:816384.

doi: 10.3389/fphy.2022.816384

\section{Cherenkov Radiation-Based Coincidence Time Resolution Measurements in BGO Scintillators}

\author{
Andrea Gonzalez-Montoro ${ }^{1}$, Shirin Pourashraf ${ }^{1}$, Joshua W. Cates ${ }^{2}$ and Craig S. Levin ${ }^{1,3,4,5 *}$ \\ ${ }^{1}$ Department of Radiology, Molecular Imaging Program at Stanford University, Stanford, CA, United States, ${ }^{2}$ Applied Nuclear \\ Physics Program, Lawrence Berkeley National Laboratory, Berkeley, CA, United States, ${ }^{3}$ Department of Physics, Stanford \\ University, Stanford, CA, United States, ${ }^{4}$ Department of Electrical Engineering, Stanford University, Stanford, CA, United States, \\ ${ }^{5}$ Department of Bioengineering, Stanford University, Stanford, CA, United States
}

Bismuth germanate oxide (BGO) scintillators can be re-introduced in time-of-flight positron emission tomography (TOF-PET) by exploiting the Cherenkov luminescence emitted as a result from $511 \mathrm{keV}$ interactions. Accessing the timing information from the relatively few emitted Cherenkov photons is now possible due to the recent improvements in enhanced near-ultraviolet high-density (NUV-HD) silicon photomultiplier (SiPM) technology, fast and low noise readout electronics, and the development of efficient data post-processing methods. In this work, we aim to develop a scalable detector element able to achieve excellent coincidence time resolution (CTR) required for TOF-PET using BGO scintillator elements of various lengths. The proposed detector element is optically coupled to $3.14 \times$ $3.14 \mathrm{~mm}^{2}$ NUV-sensitive SiPMs mounted on a custom design circuit board. In particular, we have evaluated the CTR performance of BGO crystal elements of dimensions $3 \times 3 \times$ $3 \mathrm{~mm}^{3}, 3 \times 3 \times 5 \mathrm{~mm}^{3}, 3 \times 3 \times 10 \mathrm{~mm}^{3}$, and $3 \times 3 \times 15 \mathrm{~mm}^{3}$, with chemically etched surfaces and wrapped in Teflon tape. To achieve excellent CTR performance, we apply state-of-the-art post-processing methods during data analysis. Best values of $156 \pm 6 \mathrm{ps}$, $188 \pm 5$ ps, $228 \pm 8$ ps, and $297 \pm 8$ ps CTR FWHM have been achieved for the 3, 5, 10, and $15 \mathrm{~mm}$ length BGO crystals, respectively. These values improve to $105 \pm 6 \mathrm{ps}, 127 \pm$ 8 ps, $133 \pm 4$ ps, and $189 \pm 8$ ps CTR FWHM, when only considering the Cherenkov component of the timing signal, which is extracted by considering the events with the fastest rise time (20\% of the total data). The accurate classification of the events based on their rise time is possible; thanks to the implementation of a dual threshold approach that sets the lower threshold below one light photon equivalent level and the upper one above the signal amplitude of a single photon avalanche diode (SPAD).

Keywords: positron emission tomography, time of flight, BGO scintillator, silicon photomultiplier, readout electronic, coincidence time resolution (CTR), end readout

\section{INTRODUCTION}

Bismuth germanate oxide $\left(\mathrm{Bi}_{4} \mathrm{Ge}_{3} \mathrm{O}_{12}\right)$, commonly referred as $\mathrm{BGO}$, was the scintillator of choice during the first few decades of mass commercial production of PET scanners until the late 1990s [1]. BGO scintillators are of interest in PET due to its short $511 \mathrm{keV}$ photon attenuation length $(1.1 \mathrm{~cm})$, its high-effective atomic number $\left(Z_{\text {eff }}=74\right)$, and density $\left(\rho=7.13 \mathrm{~g} / \mathrm{cm}^{3}\right)$ [2]. Moreover, BGO crystals are rather easy to grow which reduces manufacturing costs, especially when compared to 
lutetium-based fast scintillators such as cerium-doped lutetium oxy-orthosilicate $\left[\mathrm{Lu}_{2} \mathrm{SiO}_{5}(\mathrm{Ce})\right.$ or $\left.\mathrm{LSO}(\mathrm{Ce})\right]$ and lutetiumyttrium oxy-orthosilicate $\left[\mathrm{Lu}_{1.8} \mathrm{Y}_{0.2} \mathrm{SiO}_{5}(\mathrm{Ce})\right.$ or $\left.\mathrm{LYSO}(\mathrm{Ce})\right]$. These properties make BGO scintillators good candidates for building PET systems requiring a high volume of scintillation material such as total body PET scanners [3].

However, BGO scintillators exhibit a low overall scintillation light yield of 10 photons $/ \mathrm{keV}$ and long decay times $\sim 45.8 \mathrm{~ns}$ (8 $\%)-365 \mathrm{~ns}(92 \%) \mathrm{ns}$ in comparison to the 32 photons $/ \mathrm{keV}$ and $<40 \mathrm{~ns}$ for lutetium-based scintillators [4, 5]. These poor parameters comparatively discouraged investigators, and BGObased PET scanners were replaced by the lutetium-based detector design to enable TOF-PET and improved energy resolution to advance achievable noise-equivalent count rate and hence image quality [6]. A potential approach to boost the CTR of BGO for TOF-PET may be to exploit the small population of promptly emitted light photons that are generated much faster than the scintillator's dominant luminescence yield as a result of $511 \mathrm{keV}$ photon interactions [7]. An example of these prompt signatures is Cherenkov light $[7,8]$, produced when an electron in the crystal lattice is ejected with enough kinetic energy to travel faster than the speed of light in scintillating media [9]. Its detection is of interest for TOF-PET based on BGO, since relative to luminescence processes that have tens-to-hundreds of nanoseconds (ns) intrinsic decay time constants (e.g., $300 \mathrm{~ns}$ for BGO); this process is essentially instantaneous [9]. Therefore, this promptly emitted form of light can be used to derive an estimate for $511 \mathrm{keV}$ photon interaction time with lower temporal variance than achievable with the overall luminescence yield, thus improving coincidence time resolution [4].

Among the interesting Cherenkov emitter candidates to explore, BGO is one of them; due to its high index of refraction, the $511 \mathrm{keV}$ photoelectrons produce more Cherenkov light than lutetium-based scintillators $(\sim 20$ above BGO's self-absorption band and $\sim 9$ above LSO's selfabsorption band [10], and its $\sim 300 \mathrm{~nm}$ absorption band also allows it to be more transmissive to UV light [11]). An important milestone achieved to exploit Cherenkov light detection was the recent improvement of the photodetector technology such as silicon photomultipliers (SiPMs) with good photon detection efficiency (PDE) in the near-ultraviolet and low single photon time resolution (SPTR) values combined with fast, low noise readout electronics [12-14]. However, optimizing CTR from detectors that exploit the small population of prompt signatures to estimate the photon arrival time also requires special data post-processing methods.

The electronic readout has to mitigate the influence of electronic noise on achievable timing performance, which is especially difficult owing to the effective device capacitance $[15,16]$ of large area (e.g. $\left.3 \times 3 \mathrm{~mm}^{2}\right) \mathrm{SiPMs}$, in order to improve single photon response shape and SPTR [14, 17]. Practical solutions based on using low-noise, high-frequency, high-gain electronics have been already proposed to exploit the prompt Cherenkov component of the timing signal and enhance the extraction of the fast Cherenkov component in the BGO's signal, such as the so-called bootstrapping technique, which requires the use of active components [15], or passive techniques that employ a balun transformer to provide a fast path for readout that comes with gain as well as beneficial effects of common mode noise rejection [4]. Following this approach in combination with large area FBK NUV-HD SiPMs [18], values of $<100$ ps FWHM SPTR and $<300$ ps CTR for $15 \mathrm{~mm}$ long BGO scintillators were reported [4, 19], demonstrating that specific electronic readout techniques combined with UV-sensitive SiPMs are a promising approach for improving CTR with BGO-based detectors. Regarding data post-processing, the analysis should account for the high fluctuations in the number of detected Cherenkov photons [5] as well as for contamination by the slow component of the scintillation yield. As a result of fluctuations in the number of detected Cherenkov photons, long tails appear in the $511 \mathrm{keV}$ photon pair time difference (e.g., the coincidence time difference) histogram, requiring a modified Gaussian function to properly fit the data and estimate the CTR [19]. To reduce the magnitude of these tails and to more precisely determine the $511 \mathrm{keV}$ photon arrival time, a discrimination between events with high and low numbers of photons, low being from Cherenkov, and high being from the slow component of the luminescence, is necessary, as detailed in $[19,20]$. In addition, the timing signal crosses the leading-edge threshold at different levels depending on the density of Cherenkov photons detected, causing time walk effects [20]. While these tails cannot be completely removed, recent studies have demonstrated the improvement achieved using those methods; in [20], the authors were able to achieve 261 ps CTR for $3 \times 3 \times 20 \mathrm{~mm}^{3}$ BGO scintillators in combination with a high-frequency readout [21], after applying time walk corrections. Moreover, they show CTR values in the range of 205-302 ps FWHM depending on the Cherenkov yield range. Other recent studies have also shown promising progress toward using BGO in TOF-PET detectors but using digital SiPMs instead of analog ones [22]. In [10], the authors used the Philips DPC3200 digital SiPMs [23], which allow them to provide an individual timestamp from the first detected light photon (above noise) yielding 400 ps FWHM CTR for $20 \mathrm{~mm}$ length BGO crystals at room temperature and without applying any correction. These studies, among other, have demonstrated the feasibility to develop TOF-capable PET detector modules that employ a mixture of Cherenkov and slow component luminescence photons by using existing photosensor technologies coupled to $\mathrm{BGO}$ crystals with lengths that provide adequate photon detection efficiency for clinical TOFPET [24].

Following this trend, we have developed a scalable readout electronics board that consists of an improved version of the lownoise, high-frequency, and high-gain circuit presented in [4], combined using $3.14 \times 3.14 \mathrm{~mm}^{2}$ NUV-sensitive Broadcom AFBR-S4N33C013 SiPMs [25]. We have evaluated the CTR performance of BGO crystal elements of dimensions $3 \times 3 \times$ $3 \mathrm{~mm}^{3}, 3 \times 3 \times 5 \mathrm{~mm}^{3}, 3 \times 3 \times 10 \mathrm{~mm}^{3}$, and $3 \times 3 \times 15 \mathrm{~mm}^{3}$, with chemically etched surfaces and wrapped with Teflon tape, both before and after applying state-of-the-art post-processing correction methods. Promising CTR performance of $156 \pm 6$, $188 \pm 5,228 \pm 8$, and $297 \pm 8$ ps CTR FWHM have been achieved 

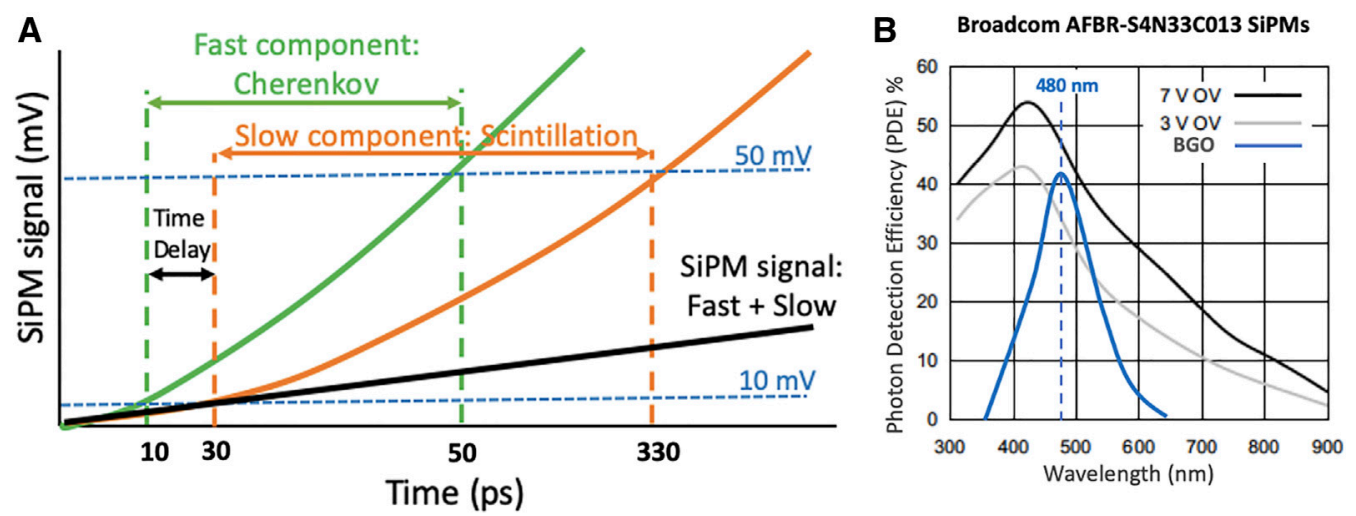

FIGURE 1 | (A). BGO's temporal components, the green line represents the fast decay component (45-60 ns) which corresponds to the emitted Cherenkov photons, and the orange line represents the slow decay component (300-365 ns) of the scintillation luminescence (original plot extracted from [20]). (B). Photon detection efficiency for the Broadcom AFBR-S4N33C013 SiPMs [25]. The blue line represents the emission spectra of BGO.

for the 3, 5, 10, and $15 \mathrm{~mm}$ length BGO crystals, respectively. We believe these results will encourage TOF-PET system developers [26] looking for a low-cost solution while reporting $<300$ ps CTR. The novelty of the work presented here is the combination of the new NUV-sensitive Broadcom ${ }^{\circledR}$ SiPMs with chemically etched BGO crystals, refined fast-timing readout circuitry, and the application of rise time correction signal processing methods. Therefore, this work presents a measurement that provides a snapshot of performance in a more scalable detector configuration.

\section{MATERIALS AND METHODS}

As described below, key elements to exploit the Cherenkov luminescence contribution for more precise CTR are the scintillation material, the photosensor technology, the electronics readout circuit, and signal processing correction methods.

\subsection{Scintillator Material: BGO}

The Cherenkov emission is produced following a $511 \mathrm{keV}$ photoelectric or Compton interaction, over a short time interval of $\mathrm{H} 10 \mathrm{ps}[27]$. As a result of the primary photoelectric or Compton interactions, electrons are emitted non-isotropically. Along the path of those energetic electrons, Cherenkov photons may be emitted with directions determined by the kinetic energy and momentum of each electron, different than those of scintillation photons which are emitted isotropically [28]. The number of Cherenkov photons generated in the scintillation material upon the $511 \mathrm{keV}$ photon interaction can be estimated using the Frank-Tamm formula [29] and mainly depends on the index of refraction and the wavelength of the scintillator material. Among other Cherenkov emitters, BGO is a good candidate to exploit the Cherenkov photon population for TOF-PET applications.

As a result of the process explained previously, the BGO's temporal signal present two components, namely a fast decay component (45-60 ns) that represents only $8-10 \%$ of the emission and corresponds to the emitted Cherenkov photons $[28,30]$ and a slow decay component (300-365 ns) that represents $92-90 \%$ of the emission [5]; see Figure 1A. As shown in Table 1, the dominant component ( 92\%) of BGO's decay time is much larger than that for other commonly used scintillators in TOF-PET such as LSO; therefore, to achieve a comparable temporal performance in BGO-based PET scanners, it is mandatory to exploit the Cherenkov component of the luminescence $(\sim 8 \%)$ [30].

To study the influence of exploiting Cherenkov radiation in the temporal performance of a custom-designed PET detector element (see Section 2.3), we made use of four different pairs of BGO crystals. In particular, we have evaluated crystal elements of $3 \times 3 \times 3 \mathrm{~mm}^{3}, 3 \times 3 \times 5 \mathrm{~mm}^{3}, 3 \times 3 \times 10 \mathrm{~mm}^{3}$, and $3 \times 3 \times$ $15 \mathrm{~mm}^{3}$ in size, with chemically etched surfaces, wrapped in Teflon tape. The crystals were coupled to a single $3.14 \times 3.14 \mathrm{~mm}^{2}$ $\mathrm{SiPM}$ in an end-readout configuration (the $3 \times 3 \mathrm{~mm}^{2}$ area was in contact with the SiPM) by means of optical grease (BC-360, Saint Gobain [31]).

\subsection{Photosensor: Near-Ultraviolet-Enhanced Silicon Photomultipliers}

The Cherenkov photon emission upon $511 \mathrm{keV}$ photon interaction in $\mathrm{BGO}$ is dominantly in the ultraviolet (UV) portion of the electromagnetic spectrum, dropping off at higher wavelengths, specifically between 310 and $850 \mathrm{~nm}$ [5]. Therefore, it is convenient to employ photosensor with enhanced photon detection efficiency (PDE) in the UV range. Previous works have evaluated the FBK NUV-HD SiPMs [18] in combination with high-frequency [22], high-gain, and lownoise readouts [4, 5] and reported CTR values below $300 \mathrm{ps}$ for long (15-20 mm) BGO crystals. In [20], the authors compared the performance of different SiPM vendors for Cherenkov detection, achieving best values when using the UVenhanced ones. 
TABLE 1 | Main properties of BGO, LSO, and $\mathrm{LaBr}_{3}[28,30]$.

\begin{tabular}{|c|c|c|c|c|c|c|c|}
\hline Material & $\mathbf{Z}_{\text {eff }}$ & Refractive index & $\rho\left(\mathrm{g} / \mathrm{cm}^{3}\right)$ & LY (ph/MeV) & Decay time (ns) & $\mu\left(\mathrm{cm}^{-1}\right) @ 511 \mathrm{keV}$ & Wavelength (nm) \\
\hline BGO & 74 & 2.15 & 7.13 & 9,000 & 45.8 (8 \%)-365 (92 \%) & 0.95 & 480 \\
\hline LSO & 63 & 1.82 & 7.4 & 29000 & 40 & 0.86 & 420 \\
\hline $\mathrm{LaBr}_{3}$ & 46.9 & 1.9 & 5.06 & 42500 & 16 & 0.476 & 380 \\
\hline
\end{tabular}
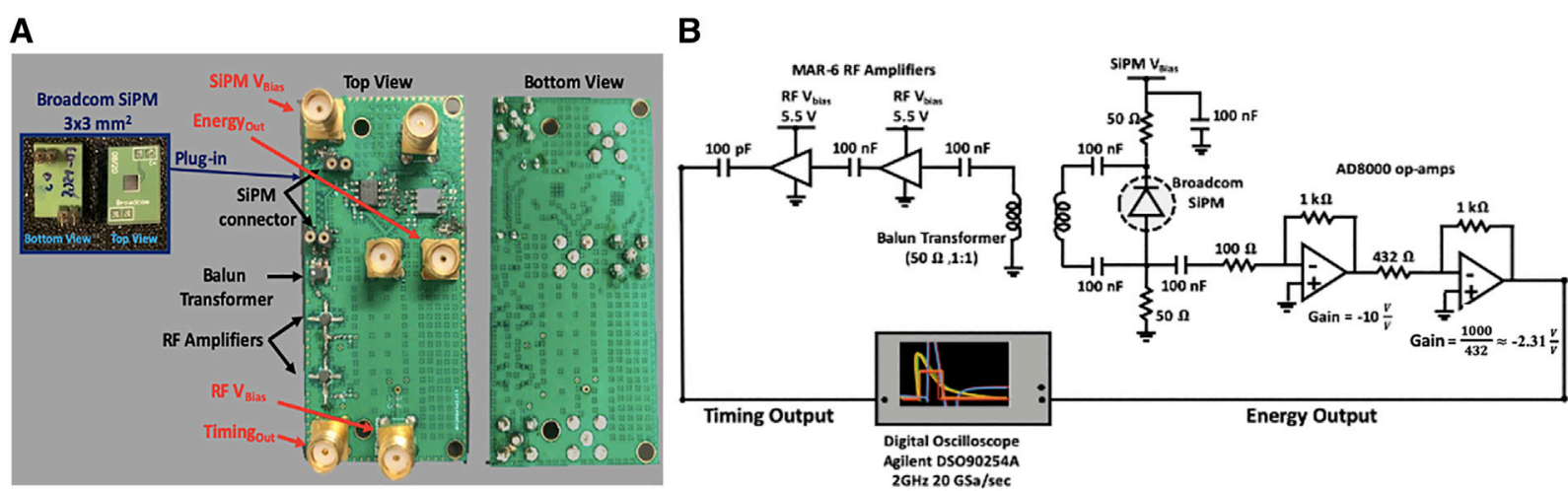

FIGURE 2 | (A). Photograph of the custom-designed board implementing the low-noise, high-speed electronics configuration and the Broadcom SiPM. (B). Circuit schematics.

For the experiments reported in this work, we have decided to use the Broadcom ${ }^{\circledR}$ AFBR-S4N33C013 SiPMs [25], since they are commercially available and can perform ultra-sensitive precision measurement of single photons in the UV range (PDE $>54 \%$ at $420 \mathrm{~nm}$ ). This high performance in the UV range is possible because of the SiPM protective layer that is made by glass that is highly transparent down to UV wavelengths which results in a broad response in the visible light spectrum with high sensitivity toward blue- and near-UV region of the light spectrum; see Figure 1B. The active area of the evaluated SiPMs is $3.14 \times$ $3.14 \mathrm{~mm}^{2}$ with a cell pitch of $30 \times 30 \mu \mathrm{m}^{2}$, and they achieved a high packing density by using through-silicon-via (TSV) technology and a chip-sized package (CSP).

\subsection{Electronics Readout Circuit}

In addition to the selection of the scintillator and SiPM technology, exploiting the Cherenkov yield to precisely estimate the photon time of interaction presents two main challenges: 1) the CTR is quite sensitive to SPTR of the SiPM and associated electronic readout used [8], and 2) the optimum leading-edge threshold should be set below one light photon equivalent level [32], where the lowest achievable threshold above the noise floor yields optimum CTR.

To account for this, we have designed a printed circuit board (PCB) that consists of a passive capacitance compensation circuit [15]. To provide an optimum timing signal, a balun transformer Macom MABA-007159 (MACOM [33]) (50 $\Omega$ impedance and 1: 1 turn ratio) is connected between the cathode and anode of the $\mathrm{SiPM}$ in a balanced-to-unbalanced configuration (which results in a 2 -fold pulse amplitude increase) to two RF amplifiers
(Minicircuits MAR-6 RF amplifiers [34]) in cascade [14]. Both the selection of the amplifiers and the trimming of their bias voltage were carefully studied until reaching optimum performance at $5.5 \mathrm{~V}$. This is a novel key feature of our design since using low voltage reduces the signal noise level and allows us to achieve good CTR performance [21] and eases the scaling up of our detector prototype, thus reducing the required power consumption. For the energy signal, the SiPM anode was connected to two AD800 operational amplifiers. Figures 2A,B show a photograph of our custom-designed board, the Broadcom SiPM, and the circuit schematics. As it can be seen, two different output signals, energy and timing, are provided. For data acquisition, raw waveforms from the detectors were acquired using a digital oscilloscope (Agilent DSO90254A 2GHz 20 $\mathrm{GSa} / \mathrm{sec})$.

\subsection{Data Acquisition and Analysis: Physical Considerations}

For data acquisition, two identical detector elements, each consisting of one $3.14 \times 3.14 \mathrm{~mm}^{2} \mathrm{SiPM}$ mounted on our custom-designed board, were used. Coincidence data were acquired by placing a ${ }^{22} \mathrm{Na}$ source $(6-\mathrm{mm}$ diameter PMMA encapsulation; $1-\mathrm{mm}$ diameter. radioactive bead; $11.8 \mu \mathrm{Ci}$ activity) between the two detectors. Using a digital oscilloscope, waveforms were acquired for each of the different crystal length pairs (four sets in total). Figure 3A displays a schematic of the experimental setup. Different SiPM bias voltages were tested to find the optimal acquisition condition. 

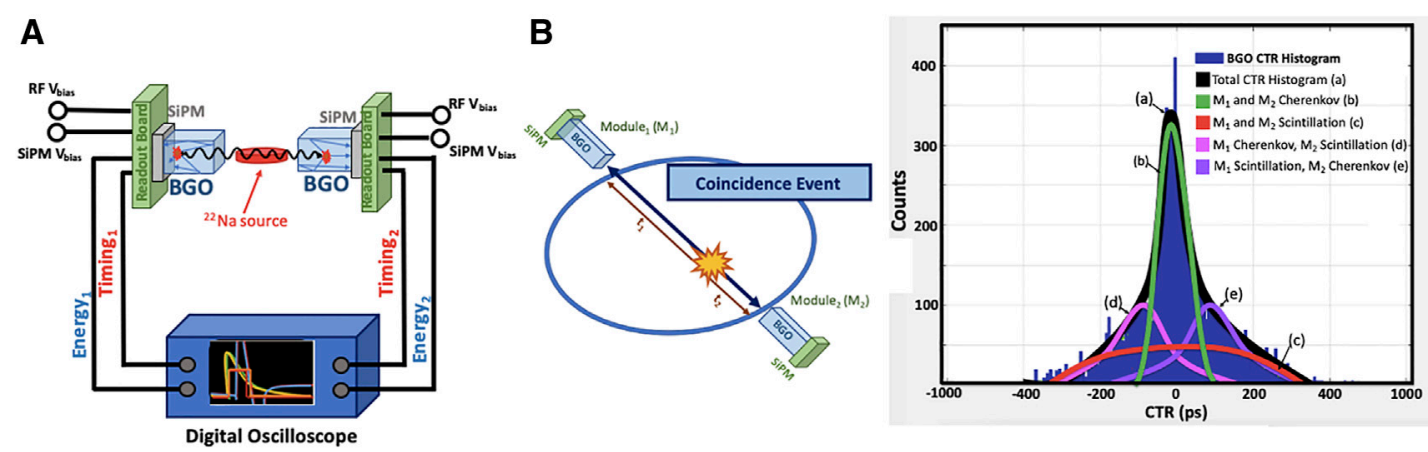

FIGURE 3 | (A). Scheme of the experimental setup (based on $3.14 \times 3.14 \mathrm{~mm}^{2}$ ) used to collect coincidence data. (B). Representative sketch of the typical coincidence time spectrum obtained for BGO scintillators showing all the different contributions $\left(M_{1}=\right.$ detector module 1 and $M_{2}=$ detector module 2).

The energy spectra for each module were estimated as the histogram of the maximum amplitude of each acquired energy waveform. The energy spectra were fitted using a Gaussian function to determine the photopeak gain as a function of the different SiPM bias voltages and crystal lengths. For data analysis, only those events falling within the FWTM of the $511 \mathrm{keV}$ photon photopeak for both detectors were processed. In addition, a leading-edge discrimination method was used to estimate time of interaction from the digitized waveforms and the influence of baseline offset from dark pulses, and cross talk events were corrected by averaging over region of the baseline in front of each pulse $[35,36]$. The energy resolution was estimated at an optimum SiPM voltage as Eres $=\Delta \boldsymbol{E}_{F W H M} / \boldsymbol{E}=2.35 \times \boldsymbol{\sigma}_{\text {phot }} / \boldsymbol{\mu}_{\text {phot }}$ for each crystal length. Note that no calibration was applied to the energy spectra.

Regarding CTR evaluation, in order to derive a more accurate estimate for $511 \mathrm{keV}$ photon time of interaction, we have followed the methods proposed in the literature $[14,19,20]$. These correction methods are applied to the experimental data; owing to the contribution of both slow and fast temporal components, BGO's coincidence time spectrum does not follow a Gaussian-like distribution as the CTR histogram shown in Figure 3B, acquired using $3 \times 3 \times 3 \mathrm{~mm}^{3}$ BGO crystals, and the plot includes drawings of each component (as explained in the following) forming the CTR histogram overlapped, with the measured data. The tails shown in the distribution arise from events where one or both detectors do not detect Cherenkov photons, but instead detect photons from the slow scintillation process. For very short crystals, the percentage of coincidence events where both detectors detect at least one Cherenkov photon is $\sim 60 \%$. This is reduced to $30-40 \%$ for $15-20-\mathrm{mm}$ long crystals depending on SiPM and crystal surface finish [14]. This results in a black profile CTR 1) distribution in Figure 3B with four components: 2) at least one Cherenkov photon in both detectors (tight Gaussian distribution, green profile), 3) no Cherenkov photons in either detector (wide Gaussian distribution from only luminescence, red profile), 4) at least one Cherenkov photon in detector $M_{1}$ (Gaussian distribution with a long tail on one side, pink profile), and 5) at least one Cherenkov photon in detector $\mathrm{M}_{2}$ (Gaussian distribution with a long tail on the other side, purple profile).
The combination of the different coincidence types is also reflected on the rise time profiles, see Figure 4A. Usually, the rise time histogram follows a centered Gaussian distribution; in this case, however, as described in Section 2.1, the BGO's luminescence signal has different temporal components, modifying the histogram. Thus, to exploit the Cherenkov contribution and achieve good CTR using BGO scintillators, three methods are included for correcting the coincidence time spectrum:

1. The rise time calculation by setting the lower threshold below one light photon equivalent threshold and the upper threshold above the signal amplitude of a single SPAD, instead of using the traditional method in which the signal rise time is estimated as the slope of the exponential fitting applied to the $10-90 \%$ of the signal [4].

2. The time delay correction method based on the different rise times observed (Fast $=$ Cherenkov; Slow $=$ standard scintillation). The method is applied as described in [19, 20]: first, the time walk $\left(T W\left(R T_{M 1}, R T_{M 2}\right)\right)$ between the two detector modules, namely $\mathrm{M}_{1}$ and $\mathrm{M}_{2}$ is calculated as the temporal difference between the timestamps of the two coincidence detected events and then is plotted versus the signal rise time (RT), measured using the methodology described previously. An example for the $T W\left(R T_{M 1}, R T_{M 2}\right)$ vs. RT scatter plot for detector $\mathrm{M}_{1}$ is shown in Figure 4B; then, a linear fit is applied to the distribution, and the fitting coefficients $\left(p_{i, n}\right)$ are used for correcting each individual event $(i)$ as follows:

$$
\begin{aligned}
\widehat{t}_{i} & =t_{i}-T W\left(R T_{M 1}, R T_{M 2}\right) \\
& =t_{i}-0.5 \cdot\left(p_{0, M 1}+p_{1, M 1} \cdot R T_{M 1}+p_{0, M 2}+p_{1, M 2} \cdot R T_{M 2}\right) .
\end{aligned}
$$

3. The classification correction method based on splitting the events into five regions for each detector based on their rise time. The events are first sorted based on their rise time and then classified into five groups containing one of the $20 \%$ of the data, i.e., the fastest $20 \%$ events are for region 1, the second fastest $20 \%$ for region 2, and subsequently for the other regions, see Figure 4A bottom for an example of the different splitting regions. Different regions other than five regions per detector 
A

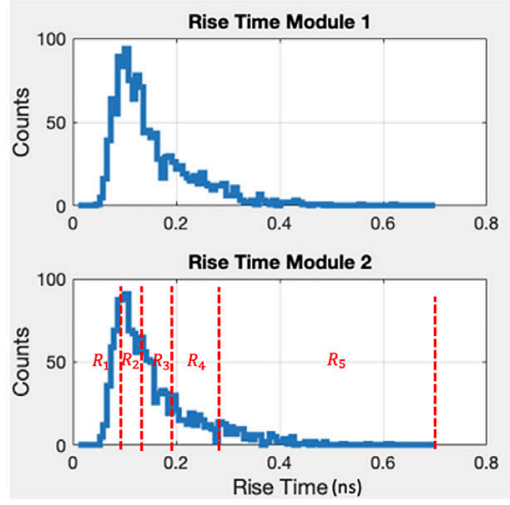

B

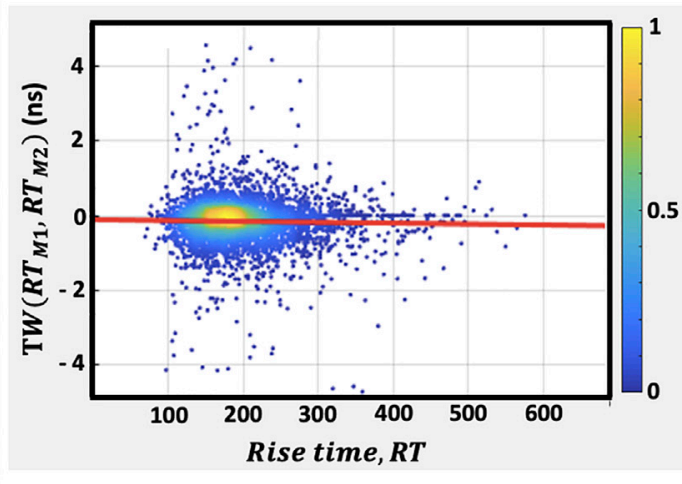

FIGURE 4 | (A). Measured rise time histograms for $3 \times 3 \times 3 \mathrm{~mm}^{3} \mathrm{BGO}$ crystals. The bottom histogram shows an example of the data splitting into five different regions (each containing $20 \%$ of the events), $R_{i}=i^{\text {th }}$ region. (B). Example for the $T W\left(R T_{M 1}, R T_{M 2}\right) v s$. RT scatter plot for $\mathrm{M}_{1}$; the solid red line depicts the linear fitting to the distribution for estimating the fitting coefficients.

could be identified, but this data sampling was selected as the best trade-off between acquired statistics and correction impact; note that these regions do not represent the different categories of coincidence types as identified in the CTR histogram of Figure 3A, where each region contains indeed both Cherenkov and scintillation event and, for this reason, each of the categories $(k=25)$ is fitted using a double Gaussian as described in [19]:

$$
\begin{aligned}
k_{\text {fit }}= & \frac{A \cdot r_{\text {Fast }}}{\sigma_{\text {Fast }}} \cdot \exp \left[-\frac{(x-\mu)^{2}}{2 \sigma_{\text {Fast }}^{2}}\right]+\frac{A \cdot\left(1-r_{\text {Fast }}\right)}{\sigma_{\text {Slow }}} \cdot[\exp [ \\
& \left.-\frac{\left(x-\mu-\mu_{\text {asim }}\right)^{2}}{2 \sigma_{\text {Slow }}^{2}}\right]
\end{aligned}
$$

where $A$ is a normalization factor which depends on the acquired statistics and bin size, $r_{\text {Fast }}$ represents the abundance of Cherenkov photons, and $\mu$ is the center of the coincidence time distribution. Note that the term $\mu_{\text {asim }}$ is zero, if the number of detected Cherenkov photons is the same for both detector modules. Finally, the time bias for each category was corrected using the centroid of the fast component of the distribution as:

$$
\hat{t}_{l}^{k}=t_{i}^{k}-\mu^{k}
$$

The corrected coincidence time spectrum $\left(\hat{t}_{l}^{k}\right)$ was then fitted using the described double Gaussian function which provided the CTR based on only Cherenkov or only scintillation photons as well as the convolution of both (total CTR value). Note that these corrections reduce the magnitude of the BGO coincidence distribution's long tails, but they do not remove them completely. Thus, the coincidence time spectra will still have non-Gaussian tails for crystals $>5 \mathrm{~mm}$ long. The tails cannot be completely removed until the Cherenkov detection efficiency is improved.

We applied all three methods to the acquired data during the post-processing. This step was carried out using the custom
MATLAB code for each on the four tested BGO crystal pairs $\left(3 \times 3 \times 3 \mathrm{~mm}^{3}, 3 \times 3 \times 5 \mathrm{~mm}^{3}, 3 \times 3 \times 10 \mathrm{~mm}^{3}\right.$, and $3 \times 3 \times$ $15 \mathrm{~mm}^{3}$ ), and the CTR was estimated as the FWHM of the corrected coincidence time distributions. The reported errors were estimated as the error provided by the fittings. Note that none of these three correction approaches reduce counts (i.e., the sensitivity) by filtering out events.

\section{RESULTS}

Figure 5A shows an example of the energy histogram for one of the $3 \times 3 \times 3 \mathrm{~mm}^{3}$ BGO crystals observed using the digital scope set in singles mode. Both the first $(511 \mathrm{keV})$ and the second $(1,274 \mathrm{keV}){ }^{22} \mathrm{Na}$ peaks are clearly resolved. Figure $5 \mathbf{B}$ shows both the energy and timing signals acquired for two coincidence detectors comprising $3 \times 3 \times 3 \mathrm{~mm}^{3}$ BGO crystals. Note that due to the low-noise level of our high-speed custom-designed electronic readout combined with enhanced UV Broadcom SiPMs, the acquired timing signals are very clean and fast which may lead to good CTR and a better discrimination of the fast and slow luminescence components.

\subsection{Photopeak Relative Gain}

Figure 6A shows the photopeak relative gain as a function of the evaluated SiPM-biasing voltage for each pair of BGO crystals. Note that the photopeak relative gain has been estimated as the ratio between each measured gain and the maximum value (provided by the $3 \times 3 \times 3 \mathrm{~mm}^{3}$ BGO crystals at $37,5 \mathrm{~V}$ ). The reported values have been calculated as the average of the two coincidence detector modules $\left(\mathrm{M}_{1}\right.$ and $\left.\mathrm{M}_{2}\right)$ used to acquire coincidence data. The standard deviation of the measured photopeak gain between the two coincidence detector elements was below 0.07 for all cases.

The energy resolution was calculated at optimum SiPM voltage being $13 \pm 2,15 \pm 3,17 \pm 3$, and $19 \pm 3 \%$ for the 3,5 , 

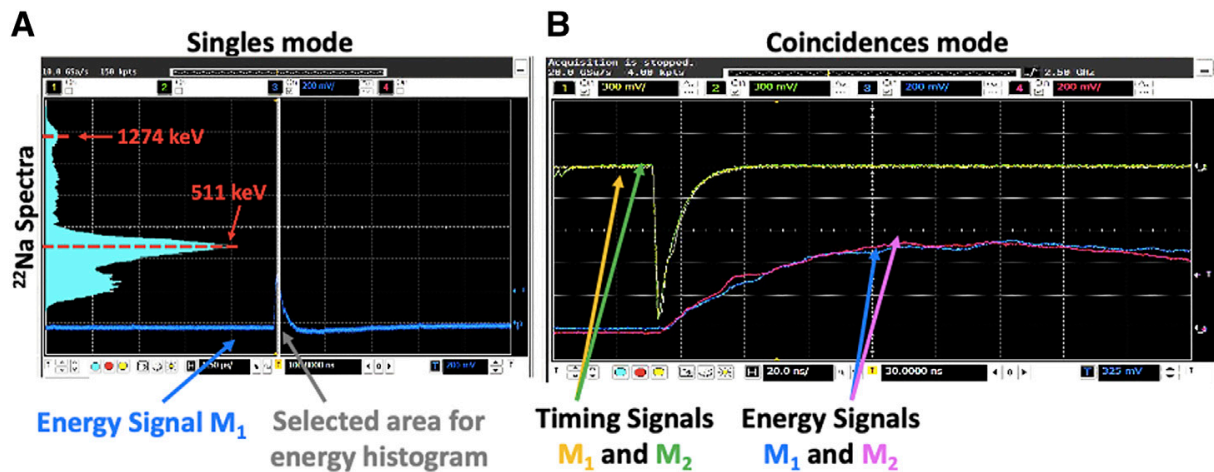

FIGURE 5 | (A). Energy histogram acquired using the digital scope in singles mode using one of the $3 \times 3 \times 3 \mathrm{~mm}^{3}$ BGO crystals. (B). Energy and timing signals acquired for two coincidence detector elements comprising $3 \times 3 \times 3 \mathrm{~mm}^{3}$ BGO crystals.
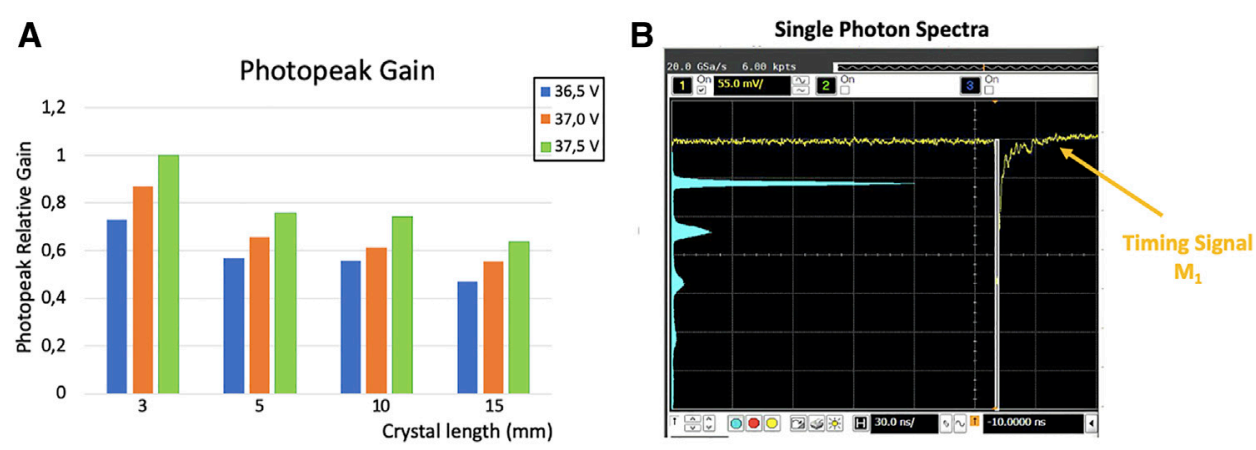

FIGURE 6 | (A). Photopeak relative gain as a function of the evaluated SiPM-biasing voltage for each of the four pairs of BGO crystals. (B). Measured single-photon spectra for $3 \times 3 \times 3 \mathrm{~mm}^{3} \mathrm{BGO}$ crystals coupled to our high-speed, low-noise custom-designed board. The histogram of $\mathrm{x}$ - and $y$-axis represents the time ( 30 ns scale) and signal level (55 $\mathrm{mV}$ scale), respectively.
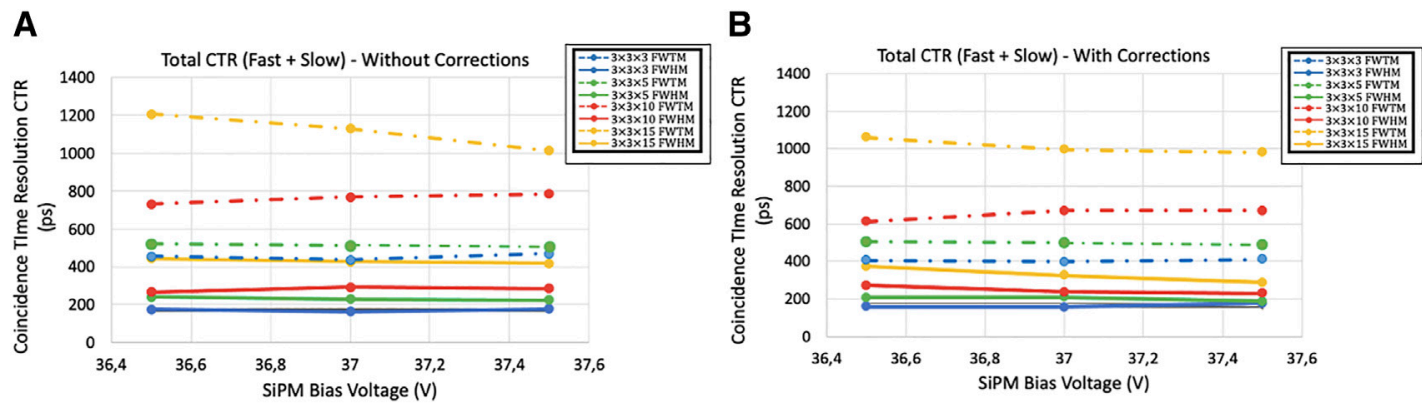

FIGURE 7 | (A, B). FWHM (solid lines) and FWTM (dashed lines) CTR values achieved as a function of the SiPM bias voltage for each of the different evaluated BGO crystal pairs before and after applying the corrections, respectively.

10 , and $15 \mathrm{~mm}$ long $\mathrm{BGO}$, respectively. Note that the energy spectra were not calibrated, but the ratio between the second and first peaks was 2,2 $\pm 0,1$ (theoretical value 1,274/411-H2.5) meaning that this is an acceptable estimation.

\subsection{CTR Performance}

Figure 6B shows the single-photon spectra measured using $3 \times$ $3 \times 3 \mathrm{~mm}^{3}$ BGO crystals coupled to our high-speed, low-noise custom-designed board. As a result, four peaks were clearly 
TABLE 2 | Best FWHM and FWTM CTR values and Cherenkov ratios obtained for each different crystal length before and after corrections.

\begin{tabular}{|c|c|c|c|c|c|c|}
\hline \multirow[t]{3}{*}{ Crystal dimension $\left(\mathrm{mm}^{3}\right)$} & \multirow{2}{*}{\multicolumn{2}{|c|}{$\begin{array}{c}\text { CTR (ps) } \\
\text { (Non-corrected) }\end{array}$}} & \multirow{2}{*}{\multicolumn{2}{|c|}{$\begin{array}{c}\text { CTR (ps) } \\
\text { (Corrected) }\end{array}$}} & \multirow[t]{3}{*}{ Cherenkov ratio (\%) } & \multirow[t]{3}{*}{ SiPM bias $(\mathrm{V})$} \\
\hline & & & & & & \\
\hline & FWHM & FWTM & FWHM & FWTM & & \\
\hline $3 \times 3 \times 3$ & $163 \pm 8$ & $437 \pm 6$ & $156 \pm 6$ & $398 \pm 9$ & 40.4 & 37 \\
\hline $3 \times 3 \times 5$ & $224 \pm 8$ & $507 \pm 10$ & $188 \pm 5$ & $589 \pm 5$ & 37.1 & 37.5 \\
\hline $3 \times 3 \times 10$ & $266 \pm 9$ & $731 \pm 8$ & $228 \pm 8$ & $613 \pm 9$ & 32.8 & 37.5 \\
\hline $3 \times 3 \times 15$ & $428 \pm 8$ & $1,013 \pm 8$ & $297 \pm 8$ & $981 \pm 11$ & 28.4 & 37.5 \\
\hline
\end{tabular}

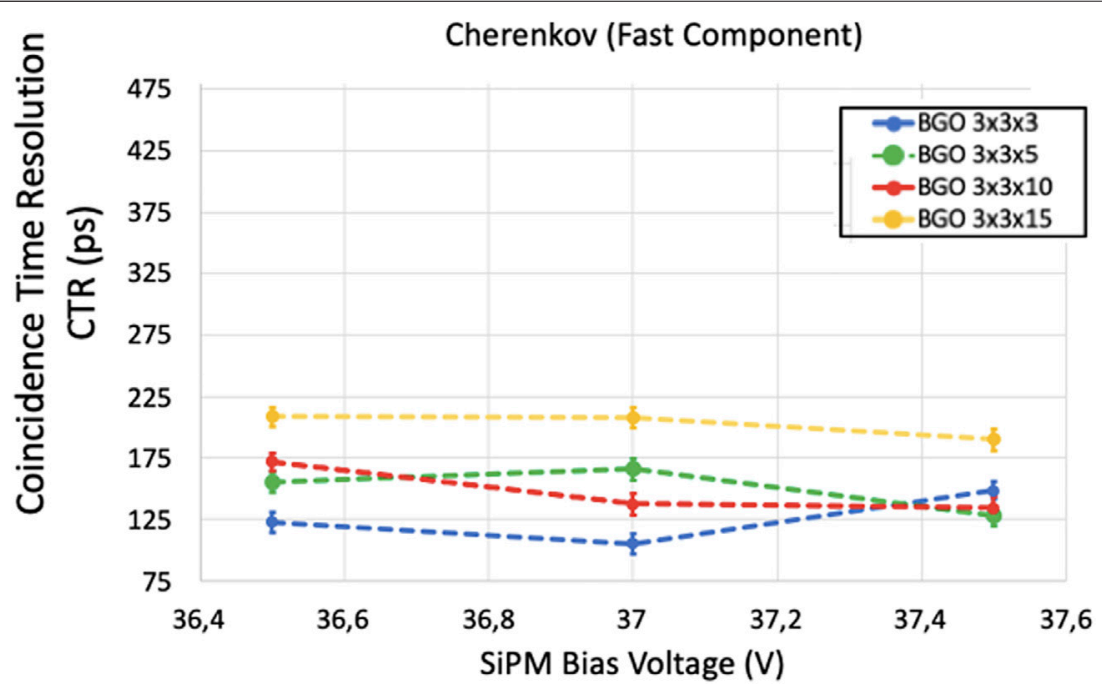

FIGURE 8|FWHM CTR values achieved when only considering the fast (Cherenkov) component of the timing signal, as a function of the SiPM bias voltage for each crystal length after applying both delay and classification corrections.

distinguished suggesting that good temporal resolution may be achieved.

Figures 7A,B depict the CTR FWHM (solid lines) and fullwidth-tenth-maximum (FWTM) (dashed lines) values achieved as a function of the SiPM bias voltage for each of the different evaluated BGO crystal pairs before and after applying the corrections outlined in Section 2.4, respectively.

Before applying any correction method, best CTR FWHM values of $163 \pm 8,224 \pm 8,266 \pm 9$, and $428 \pm 8$ ps and best CTR FWTM values of $437 \pm 6,507 \pm 10,731 \pm 8$, and $1,013 \pm 8$ ps have been obtained for $3 \times 3 \times 3 \mathrm{~mm}^{3}, 3 \times 3 \times 5 \mathrm{~mm}^{3}, 3 \times 3 \times 10 \mathrm{~mm}^{3}$, and $3 \times 3 \times 15 \mathrm{~mm}^{3}$ BGO crystals, respectively. After applying both the delay and classification methods, these values improved to $156 \pm 6,188 \pm 5,228 \pm 8$, and $297 \pm 8$ ps for CTR FWHM and $398 \pm 9,489 \pm 5,613 \pm 9$, and $981 \pm 11$ ps for CTR FWTM for $3 \times$ $3 \times 3 \mathrm{~mm}^{3}, 3 \times 3 \times 5 \mathrm{~mm}^{3}, 3 \times 3 \times 10 \mathrm{~mm}^{3}$, and $3 \times 3 \times 15 \mathrm{~mm}^{3}$ BGO crystals, respectively. Quantitatively, this implies CTR improvements of $3.2,6.7,8.1$, and $12.6 \%$, respectively. The ratio of events where both sensors detect at least one Cherenkov photon was also estimated for each of the BGO crystal lengths reporting, with the corresponding ratios of 40.4, $37.1,32.8$, and $28.4 \%$ for the $3,5,10$, and $15 \mathrm{~mm}$ long crystals, respectively. Table 2 reports the best CTR values (FWHM and FWTM) and Cherenkov ratios for each crystal length before and after corrections. Note that the values presented in Table 2 have been estimated after merging all the events (Cherenkov + Scintillation), and thus any coincidence was discarded. In other words, the fitting considers the convolution of the fast (Cherenkov) and slow (Scintillation) timing components.

Figure 8 depicts the CTR values achieved when only considering the fast (Cherenkov) component of the BGO's timing signal as a function of the SiPM bias voltage for each crystal length and after applying the previously described corrections. Best CTR FWHM values of $105 \pm 6,127 \pm 8$, $133 \pm 4$, and $189 \pm 8$ ps have been reported for $3 \times 3 \times 3 \mathrm{~mm}^{3}$, $3 \times 3 \times 5 \mathrm{~mm}^{3}, 3 \times 3 \times 10 \mathrm{~mm}^{3}$, and $3 \times 3 \times 15 \mathrm{~mm}^{3}$ BGO crystals, respectively. Table 3 summarizes the FWHM CTR results. Note that the second and third columns report the CRT values when only considering the fast (Cherenkov contribution) or slow (Scintillation contribution) component of the timing signal while the fourth and fifth columns report the CTR values after merging all the events (Cherenkov + Scintillation) again, and thus any coincidence was discarded in these cases. 
TABLE 3 | Best FWHM CTR values obtained for each different crystal length before and after corrections. The second and third columns report the CRT values when only considering the fast (Cherenkov contribution) or slow (Scintillation contribution) component of the timing signal.

\begin{tabular}{|c|c|c|c|c|c|}
\hline \multirow[t]{2}{*}{$\begin{array}{l}\text { Crystal dimension } \\
\left(\mathrm{mm}^{3}\right)\end{array}$} & $\begin{array}{c}\text { Fast CTR } \\
\text { (ps) }\end{array}$ & $\begin{array}{c}\text { Slow CTR } \\
\text { (ps) }\end{array}$ & CTR (ps) & CTR (ps) & $\begin{array}{l}\text { SiPM bias } \\
\text { (V) }\end{array}$ \\
\hline & (Cherenkov) & (Scintillation) & (Corrected) & (Non-corrected) & \\
\hline $3 \times 3 \times 3$ & $105 \pm 6$ & $229 \pm 7$ & $156 \pm 6$ & $163 \pm 8$ & 37 \\
\hline $3 \times 3 \times 5$ & $127 \pm 8$ & $382 \pm 8$ & $188 \pm 5$ & $224 \pm 8$ & 37.5 \\
\hline $3 \times 3 \times 10$ & $133 \pm 4$ & $478 \pm 6$ & $228 \pm 8$ & $266 \pm 9$ & 37.5 \\
\hline $3 \times 3 \times 15$ & $189 \pm 8$ & $800 \pm 9$ & $297 \pm 8$ & $428 \pm 8$ & 37.5 \\
\hline
\end{tabular}

\section{DISCUSSION}

The present article describes a potential scalable solution for BGO-based TOF-PET detectors. Fast and low noise front-end electronics minimize the influence of electronic noise on sensortiming response and result in the excellent signal-to-noise ratio to provide an optimum timing signal well above the noise level. This is the key to discriminate the fast component (Cherenkov) of the timing signal and exploit the small Cherenkov photon yield. An advantage of the experimental setup in the present work is the selected amplifiers and their configuration that allows optimum performance at lower voltage $(5.5 \mathrm{~V})$, thus reducing the signal noise level. The readout board integrated $3.14 \times 3.14 \mathrm{~mm}^{2}$ Broadcom $^{\oplus}$ AFBR-S4N33C013 SiPMs, which have reported ultra-sensitive precision measurement of single photons in the UV range (PDE $>54 \%$ at $420 \mathrm{~nm}$ ) [25].

For the evaluation of the detector's ability to discriminate the Cherenkov contribution, we tested BGO crystal elements of $3 \times$ $3 \times 3 \mathrm{~mm}^{3}, 3 \times 3 \times 5 \mathrm{~mm}^{3}, 3 \times 3 \times 10 \mathrm{~mm}^{3}$, and $3 \times 3 \times 15 \mathrm{~mm}^{3}$ in size, with chemically etched surfaces and wrapped using Teflon tape. The reason behind using this metric is that $15-\mathrm{mm}$ long BGO crystals have $\sim 6 \%$ higher photoelectric absorption probability for $511 \mathrm{keV}$ photons than $20-\mathrm{mm}$ lutetium-based crystals; thus, these results are directly relevant to the practical detector design for high detection efficiency clinical PET systems at lower cost [37].

During data analysis, the photopeak relative gain was estimated for each SiPM-biasing voltage and for each BGO crystal length (Figure 6). The standard deviations between the photopeak gain for the two modules were below 0.07 confirming the similar performance of both detectors. As expected, higher gains were achieved at higher bias voltages. Excellent discrimination of the single photon response (Figure 4) was achieved, thanks to the electronic readout design, allowing an optimum threshold for leading-edge time pickoff well below the single photon equivalent amplitude and just above baseline noise. We also implement a dual threshold approach to reduce the tailing in the coincidence time distribution, as suggested in [20]. This method helps because events that do not detect any Cherenkov photons in one or both detectors have a skew in the detection time, generating rise time histograms as the one shown in Figure 4A. The time pickoff derived only from BGO's slow luminescence results in a slightly delayed timestamp. The rise time correction and classification methods provide some correction for this delay, which in turn reduces the tails by effectively pulling in the events that have no Cherenkov photons in one or both detectors toward the center of the CTR distribution $[19,20]$. This skew correction also improves CTR as demonstrated. For the longer crystals with lower density of Cherenkov photons, this correction has a larger impact because it is correcting for skew in a greater number of coincidence events that do not have Cherenkov photons.

Using the experimental setup shown in Figure 3A, we achieved best CTR FWHM values of $163 \pm 8,224 \pm 8,266 \pm$ 9 , and $428 \pm 8$ ps and best CTR FWTM values of $437 \pm 6,507 \pm$ $10,731 \pm 8$, and $1,013 \pm 8$ ps for $3 \times 3 \times 3 \mathrm{~mm}^{3}, 3 \times 3 \times 5 \mathrm{~mm}^{3}, 3 \times$ $3 \times 10 \mathrm{~mm}^{3}$, and $3 \times 3 \times 15 \mathrm{~mm}^{3}$ BGO crystals before applying any correction (Figure 7A). These values improved to a CTR of $156 \pm 6,188 \pm 5,228 \pm 8$, and $297 \pm 8$ ps for FWHM and $398 \pm 9$, $489 \pm 5,613 \pm 9$, and $981 \pm 11$ ps for CTR FWTM, respectively, after applying the corrections described in Section 2.4 (Figure 7B), which represents an improvement of 3.2, 6.7, 8.1, and $12.6 \%$. As expected, a degradation of CTR was observed as a function of the increased crystal length due to possible missalignment and increased light transit time inside the crystal before reaching the SiPM. The achieved CTR values are already acceptable to build a Cherenkov-based PET detector comprising long BGO crystals. However, one clear area for improvement is increasing the light extraction efficiency for Cherenkov photons. One way to address this directly is to leverage the near-complete light collection efficiency available from the "side-readout" configuration [38]. The side-readout approach has been demonstrated to reduce the transit time variance of the light photons to the photosensor while enhancing the light collection efficiency. We are currently designing a board to test the side-readout configuration in our Cherenkov-based detector. In addition, the presented results were acquired at room temperature with no active cooling, and thermal regulation is likely required to mitigate the higher uncorrelated and correlated noise in a scaled, multiplexed detector configuration.

The applied methodology facilitates data corrections for $511 \mathrm{keV}$ photon time of interaction, as a function of signal rise time, which partially compensate inherent delays in timing estimators derived from events with no Cherenkov photons. When considering only the fast (Cherenkov) component of the timing signal, best values of $105 \pm 6,127 \pm 8,133 \pm 4$, and $189 \pm 8$ ps for CTR FWHM long BGO crystals have been achieved for $3 \times 3 \times 3 \mathrm{~mm}^{3}, 3 \times 3 \times 5 \mathrm{~mm}^{3}, 3 \times 3 \times 10 \mathrm{~mm}^{3}$, and $3 \times 3 \times 15 \mathrm{~mm}^{3}$ BGO crystals, respectively, as shown in Figure 8 . 
Looking at individual event categories may allow estimation of event position along each system line of response (LOR) with different precisions corresponding to the different time resolution values [19]. The standard PET image reconstruction methods allocate the same TOF kernel resolution to all events. However, since the rise time information allows classification of events, a gain in SNR is expected when each category of the events is allocated to its proper TOF kernel; thus, events having a better time resolution can provide higher TOF kernel resolution along the LOR and thus better reconstructed image SNR, while events with worse time resolution are used in addition to maintain the high sensitivity of BGO [20]. The work presented in [39] reports a simulation toolkit that introduces multiple timing spreads on the coincident events of a full cylindrical BGO-Cherenkov PET model and an image reconstruction procedure that incorporates the multi-kernel idea. The results demonstrate a better contrast-to-noise ratio for the multi-kernel BGO model.

Note that the higher photon detection efficiency of BGO offers another strong advantage. It can be estimated as the effective sensitivity gain for BGO's higher detection efficiency combined with TOF performance for the different underlying components of the CTR distribution:

$$
\begin{aligned}
\text { Ef fective Sensitivt } y_{B G O}= & (\text { TOF gain } \text { Cherenkov } \\
& \left.\cdot \text { Fractional Weight }_{\text {Cherenkov }}\right) \\
& +\left(\text { TOF gain }_{\text {Scintillation }} \cdot(1\right. \\
& \left.\left.- \text { Fractional Weight }_{\text {Cherenkov }}\right)\right) .
\end{aligned}
$$

Therefore, a $15-\mathrm{mm}$ long BGO crystal element with $40 \%$ of events having Cherenkov photons in both detectors, with fast Gaussian (Cherenkov) FWHM of $\sim 250$ ps and slow Gaussian (Scintillation) of $\sim 1 \mathrm{~ns}$ FWHM achieves the same effective sensitivity as a $20-\mathrm{mm}$ long LSO crystal element with 320 ps FWHM timing performance. So, even immediate implementations of BGO can have a significant impact. Technologies that improve Cherenkov detection efficiency can also eventually push performance toward the same effective sensitivity as a 200 -ps LSO scanner, or better.

The CTR values reported in this work are comparable or better to those achieved by state-of-the-art commercial TOF-PET systems [40, 41]. This work thus indicates to be feasible that BGO-based TOF-PET detector modules could be developed with the same effective sensitivity compared to the one achieved using 20 -mm long LSO-based detector modules that achieve $<350$ ps FWHM CTR, facilitating high performance clinical PET systems with 4-5-fold lower scintillation material cost. Furthermore, other advantages associated to BGO scintillators are shorter $511 \mathrm{keV}$ photon attenuation length $(\mathrm{BGO}=1.1 \mathrm{~cm})$ than lutetium-based ones (i.e., LSO $=1.3 \mathrm{~cm}$ ), thus less volumes of BGO material are required to stop the same amount of incoming radiation; BGO's effective atomic number $\left(\mathrm{Z}_{\text {eff }}=74\right)$ is higher than the one for lutetium-based scintillators which increases the probability of photoelectric interactions instead of Compton ones. Note that photoelectric interactions are preferred in PET since they allow for a more accurate positioning of the annihilation point within the scintillator, and BGO scintillators do not present intrinsic radiation while lutetium-based scintillators present the intrinsic radiation of ${ }^{177} \mathrm{Lu}$, thus the contributions of other decay products are not present minimizing possible pile-up or false coincidences.

As a closing remark, we would like to comment on the proposed detector design scalability. We are currently upgrading the presented standard end-readout detector architecture to large side-readout area detector modules which include six SiPMs. In this side-readout version of our design, the circuitry electronics will be integrated in a wisely designed highspeed and compact PCB board. For the accurate determination of the signal's rise time, we plan to include an ASIC configured to generate two timestamps on the leading edge of the pulse. We will follow a similar approach to the one shown in [42], in which the authors proposed a readout design for lutetium-based scintillators that allow to acquire multiple timestamps per detected event using two ASICs. In our case, the ASICs will be customized with the lower threshold set below one light photon equivalent threshold and the upper threshold above the signal amplitude of a single SPAD.

Moreover, since power consumption is a major concern when scaling up the electronic design of single detectors to compact PET modules, our circuitry has been carefully trimmed to ensure optimal performance of the components at low bias voltage (amplifier's bias voltage $5.5 \mathrm{~V}$ ). Note that low operation voltage is also the key to minimize the influence of electronic noise on sensor-timing response and thus to provide an optimum timing signal well above the noise level which is the key to discriminate the fast component (Cherenkov) of the timing signal and exploit the small Cherenkov photon yield. Finally, regarding the implementation of the correction methods based on non-Gaussian TOF kernels, we plan to apply such algorithms once the data acquisition has been completed. Acquired data will be transferred to a PC for the application of the correction methods which will be followed by image reconstruction. The inclusion of several TOF kernels in reconstruction was already demonstrated in [39], so we will follow a similar approach. However, this is out of the scope of this article and will be discussed in detail in a future work.

Summarizing, the combination of the aforementioned BGO's advantages and the good CTR performance provided by exploiting the BGO-Cherenkov component allows to achieve the same sensitivity while employing less volume of scintillation materials, thus reducing cost and enabling more compact scanners. These properties make BGO scintillators good candidates for building PET systems requiring a high volume of scintillation material such as total body PET scanners.

\section{CONCLUSION}

We have reported the CTR value for BGO crystal elements of various lengths with chemically processed surface treatments, using an optimized, fast, and high-frequency readout electronic design that minimizes the influence of electronic noise. The board was evaluated using $3.14 \times 3.14 \mathrm{~mm}^{2}$ Broadcom ${ }^{\circledR}$ AFBRS4N33C013 SiPMs. These SiPMs present enhanced 
performance in the UV region which is the key for exploiting the small Cherenkov photon population generated in BGO scintillators, thus making it feasible for their deployment in TOF-PET designs. Our data analysis included state-of-the-art data post-processing methods [20] which allowed us to achieve best CTR values of $163 \pm 8,224 \pm 8,266 \pm 9$, and $428 \pm 8$ ps for CTR FWHM and $398 \pm 9,489 \pm 5,613 \pm 9$, and $981 \pm 11$ ps for CTR FWTM for $3 \times 3 \times 3 \mathrm{~mm}^{3}, 3 \times 3 \times 5 \mathrm{~mm}^{3}, 3 \times 3 \times 10 \mathrm{~mm}^{3}$, and $3 \times 3 \times 15 \mathrm{~mm}^{3}$ BGO crystals, respectively. The results presented here indicate that fast, low-noise front-end electronics employed as readout for BGO-based PET detectors are suitable for building BGO-based PET detectors that provide CTR values equivalent to what is currently achievable in state-of-the-art TOF-PET systems (214-480 ps FWHM) [40, 41]. To our knowledge, this is the first work reporting the performance of chemically etched BGO crystals coupled by means of optical grease to the new NUV-sensitive Broadcom ${ }^{\circledR}$ SiPMs, which includes the application of rise time correction methods that allowed us to achieve improved CTR values in comparison with other state-of-the-art works. Furthermore, our prototype detector readout electronics have been trimmed, and the selection of its amplifiers was carefully studied until reaching optimum performance at $5.5 \mathrm{~V}$. This low voltage reduces the signal noise level and is the key to achieve good CTR performance while also allowing for scaling up the design.

\section{DATA AVAILABILITY STATEMENT}

The raw data supporting the conclusion of this article will be made available by the authors, without undue reservation.

\section{REFERENCES}

1. Cho ZH, Farukhi MR. Bismuth Germanate as a Potential Scintillation Detector in Positron Cameras. J Nucl Med (1977) 18(8):840-4.

2. Moses WW. Recent Advances and Future Advances in Time-Of-Flight PET. Nucl Instr Methods Phys Res Section A: Acc Spectrometers, Detectors Associated Equipment (2007) 580:919-24. doi:10.1016/j.nima.2007.06.038

3. Gonzalez-Montoro A, Sanchez F, Majewski S, Zanettini S, Benlloch JM, Gonzalez AJ. Highly Improved Operation of Monolithic BGO-PET Blocks. J Inst (2017) 12:C11027. doi:10.1088/1748-0221/12/11/c11027

4. Cates JW, Levin CS. Electronics Method to advance the Coincidence Time Resolution with Bismuth Germanate. Phys Med Biol (2019) 64:175016. doi:10. 1088/1361-6560/ab31e3

5. Gundacker S, Martinez Turtos R, Kratochwil N, Pots RH, Paganoni M, Lecoq P, et al. Experimental Time Resolution Limits of Modern SiPMs and TOF-PET Detectors Exploring Different Scintillators and Cherenkov Emission. Phys Med Biol (2020) 65:025001. doi:10.1088/1361-6560/ab63b4

6. Conti M, Bendriem B. The New Opportunities for High Resolution Clinical TOF PET. Clin Translational Imaging (2019) 7:139-47. doi:10.1007/s40336019-00316-5

7. Lecoq P. Pushing the Limits in Time-Of-Flight PET Imaging. IEEE Trans Radiat Plasma Med Sci (2017) 1:473-85. doi:10.1109/trpms.2017.2756674

8. Gundacker S, Auffray E, Pauwels K, Lecoq P. Measurement of Intrinsic Rise Times for Various L(Y)SO and LuAG Scintillators with a General Study of Prompt Photons to Achieve 10 Ps in TOF-PET. Phys Med Biol (2016) 61: 2802-37. doi:10.1088/0031-9155/61/7/2802

9. Cherenkov P. Visible Emission of Clean Liquids by Action of $\gamma$ Radiation. Dokl Akad Nauk SSSR (1934) 2:451-4.

\section{AUTHOR CONTRIBUTIONS}

AG-M, was responsible for the design of the experiments reported in this work, data collection and analysis, modification of the code used for the analysis, and manuscript writing; SP, designed and assembled the improved low-noise, fast, and high-frequency circuit board, trimmed the signals and the experimental setup, and participated during the data collection process; JC, developed the initial code used for data analysis and the first approach as reported in [4], and he also provided feedback for the design of the experiments and deep explanations about the physics underlying the processes described here; and CL, supervised all the experiments and provided valuable feedback for the interpretation of the results. It has to be mentioned that all co-authors were equally involved during the discussions concerning the experiments and the understanding of the reported results as well as in the review process of the manuscript.

\section{FUNDING}

This work was supported by NIH Grants R01CA214669 and R01EB025125. AG-M is partially supported by the VALi $+\mathrm{d}$ Program for Researchers in Postdoctoral Phase of the Ministry of Labor and Social Economy (Generalitat de Valencia) and the EU Social Fund.

\section{ACKNOWLEDGMENTS}

The authors would like to thank Stefan Brunner from Broadcom for providing the SiPM samples used in this study.

10. Brunner SE, Schaart DR. BGO as a Hybrid Scintillator / Cherenkov Radiator for Cost-Effective Time-Of-Flight PET. Phys Med Biol (2017) 62:4421-39. doi:10.1088/1361-6560/aa6a49

11. Williams PA, Rose AH, Lee KS, Conrad DC, Day GW, Hale PD. Optical, Thermo-Optic, Electro-Optic, and Photoelastic Properties of Bismuth Germanate (Bi_4Ge_3O_12). Appl Opt (1996) 35:3562-9. doi:10.1364/ao. 35.003562

12. Piemonte C, Acerbi F, Ferri A, Gola A, Paternoster G, Regazzoni V, et al. Performance of NUV-HD Silicon Photomultiplier Technology. IEEE Trans Electron Devices (2016) 63:1111-6. doi:10.1109/ted.2016.2516641

13. Kwon I, Kang T, Hammig MD. Experimental Validation of Charge-Sensitive Amplifier Configuration that Compensates for Detector Capacitance. IEEE Trans Nucl Sci (2016) 63:1202-8. doi:10.1109/tns.2016.2530065

14. Cates JW, Gundacker S, Auffray E, Lecoq P, Levin CS. Improved Single Photon Time Resolution for Analog SiPMs with Front End Readout that Reduces Influence of Electronic Noise. Phys Med Biol (2018) 63:185022. doi:10.1088/ 1361-6560/aadbcd

15. Zhang N, Schmand MJ. Bootstrapping Readout for Large Terminal Capacitance Analog-SiPM Based Time-Of-Flight PET Detector. US Patent US2016/0327657 (2016).

16. Kwon I, Kang T, Wells BT, D'Aries LJ, Hammig MD. Compensation of the Detector Capacitance Presented to Charge-Sensitive Preamplifiers Using the Miller Effect. Nucl Instr Methods Phys Res Section A: Acc Spectrometers, Detectors Associated Equipment (2015) 784:220-5. doi:10.1016/j.nima.2014. 12.049

17. Pourashraf S, Gonzalez-Montoro A, Won JY, Lee MS, Cates JW, Zhao Z, et al. Scalable Electronic Readout Design for a 100 Ps Coincidence Time Resolution TOF-PET System. Phys Med Biol (2021) 66(8):1361-6560. doi:10.1088/1361$6560 / a b f 1 b c$ 
18. Gola A, Acerbi F, Capasso M, Marcante M, Mazzi A, Paternoster G, et al. NUV-sensitive Silicon Photomultiplier Technologies Developed at Fondazione Bruno Kessler. Sensors (2019) 19(2):308. doi:10.3390/s19020308

19. Kratochwil N, Gundacker S, Lecoq P, Auffray E. Pushing Cherenkov PET with BGO via Coincidence Time Resolution Classification and Correction. Phys Med Biol (2020) 65:115004. doi:10.1088/1361-6560/ab87f9

20. Kratochwil N, Auffray E, Gundacker S. Exploring Cherenkov Emission of BGO for TOF-PET. IEEE Trans Radiat Plasma Med Sci (2021) 5:619-29. doi:10.1109/TRPMS.2020.3030483

21. Gundacker S, Turtos RM, Auffray E, Paganoni M, Lecoq P. High-frequency SiPM Readout Advances Measured Coincidence Time Resolution Limits in TOF-PET. Phys Med Biol (2019) 64(5):055012-6560. doi:10.1088/1361-6560/ aafd52

22. Gundacker S, Auffray E, Jarron P, Meyer T, Lecoq P. On the Comparison of Analog and Digital SiPM Readout in Terms of Expected Timing Performance. Nucl Inst. Methods Phys Res A (2014) 787:6-11. doi:10.1016/j.nima.2014. 10.020

23. Liu Z, Gundacker S, Pizzichemi M, Ghezzi A, Auffray E, Lecoq P, et al. Indepth Study of Single Photon Time Resolution for the Philips Digital Silicon Photomultiplier. J Inst (2016) 11:P06006. doi:10.1088/1748-0221/11/06/ p06006

24. Brunner SE, Gruber L, Marton J, Suzuki K, Hirtl A. Studies on the Cherenkov Effect for Improved Time Resolution of TOF-PET. IEEE Trans Nucl Sci (2014) 61:443-7. doi:10.1109/tns.2013.2281667

25. Broadcom ${ }^{\circledR}$. AFBR-S4N33C013 NUV-HD Single Silicon Photomultiplier Data Sheet. Available from: https:/www.broadcom.com/products/ optical-sensors/silicon-photomultiplier-sipm/afbr-s4n33c013 (Accessed January 4, 2022).

26. Alokhina M. Design of the Cherenkov TOF Whole-Body PET Scanner Using GATE Simulation. Medical Physics. Gif-sur-Yvette: Université Paris Saclay (COmUE); Kiivs kij nacional nij unìversitet imeni Tarasa Sõevcõenka Ukraine (2018).

27. Williams RT, Ucer KB, LoPresti JL. In the First Instants ... Ultrafast Views of Radiation Effects. Radiat Measurements (2001) 33:497-502. doi:10.1016/ s1350-4487(01)00041-5

28. Roncali E, Il Kwon S, II, Jan S, Berg E, Cherry SR. Cerenkov Light Transport in Scintillation Crystals Explained: Realistic Simulation with GATE. Biomed Phys Eng Express (2019) 5:035033. doi:10.1088/2057-1976/ab0f93

29. Jelley JV. Cerenkov Radiation and its Applications. Br J Appl Phys (1955) 6:227. doi:10.1088/0508-3443/6/7/301

30. Moszyński M, Gresset C, Vacher J, Odru R. Timing Properties ofBGOscintillator Nucl. Instrum Methods Phys Res (1981) 188:403-9.

31. Saint-Gobain. BC-630 Silicone Grease DATASHEET (2017). Available from: https://www.crystals.saint-gobain.com/sites/imdf.crystals.com/files/ documents/bc-630.pdf (Accessed September 11, 2019).

32. Gundacker S, Acerbi F, Auffray E, Ferri A, Gola A, Nemallapudi MV, et al. State of the Art Timing in TOF-PET Detectors with LuAG, GAGG and L(Y)SO Scintillators of Various Sizes Coupled to FBK-SiPMs. J Inst (2016) 11:P08008. doi:10.1088/1748-0221/11/08/p08008
33. MACOM. MABA-007159 Data Sheet 2019 Cdn. Available from: macom.com/ datasheets/MABA-007159-000000.pdf (Accessed January 27, 2020).

34. Minicircuits. MAR-6 RF Amplifier Data Sheet 2019. Available from: minicircuits.com/pdfs/MAR-6+.pdf (Accessed April 22, 2019).

35. Vinke R, Seifert S, Schaart DR, Schreuder FP, de Boer MR, van Dam HT, et al. Optimization of Digital Time Pickoff Methods for LaBr3-SiPM TOF-PET Detectors NSS/MIC. In: IEEE Nuclear Science Symp. Conf. Record; 2009, Oct 24-Nov 1; Orlando, FL, USA (2009). p. 2962-8.

36. Seifert S, Schaart DR. Improving the Time Resolution of TOF-PET Detectors by Double-Sided Readout. IEEE Trans Nucl Sci (2015) 62:3-11. doi:10.1109/ tns.2014.2368932

37. Humm JL, Rosenfeld A, Del Guerra A. From PET Detectors to PET Scanners. Eur J Nucl Med Mol Imaging (2003) 30:1574-97. doi:10.1007/s00259-0031266-2

38. Yeom JY, Vinke R, Levin CS. Side Readout of Long Scintillation crystal Elements with Digital SiPM for TOF-DOI PET. Med Phys (2014) 41: 122501. doi:10.1118/1.4901524

39. Efthimiou N, Kratochwil N, Gundacker S, Polesel A, Salomoni M, Auffray E, et al. TOF-PET Image Reconstruction with Multiple Timing Kernels Applied on Cherenkov Radiation in BGO IEEE. Trans Rad Plasma Med Sci (2020) 5(5): 703-11. doi:10.1109/TRPMS.2020.3048642

40. Siemens Helathcare. Biograph Vision PET/CT 2019 usa. Available from: healthcare.siemens.com/molecular-imaging/pet-ct/biograph-vision (Accessed May 6, 2019).

41. Philips. Vereos PET/CT 2019 usa. Available from: philips.com/healthcare/ product/HC882446/vereos-digital-petct-provenaccuracy-inspiresconfidence\#galleryTab=360 (Accessed July 1, 2018).

42. LaBella A, Tavernier S, Woody C, Purschke M, Zhao W, Goldan AH. Toward 100 Ps Coincidence Time Resolution Using Multiple Timestamps in DepthEncoding PET Modules: A Monte Carlo Simulation Study. IEEE Trans Radiat Plasma Med Sci (2021) 5(5):679-86. doi:10.1109/trpms.2020.3043691

Conflict of Interest: The authors declare that the research was conducted in the absence of any commercial or financial relationships that could be construed as a potential conflict of interest.

Publisher's Note: All claims expressed in this article are solely those of the authors and do not necessarily represent those of their affiliated organizations, or those of the publisher, the editors, and the reviewers. Any product that may be evaluated in this article, or claim that may be made by its manufacturer, is not guaranteed or endorsed by the publisher.

Copyright (c) 2022 Gonzalez-Montoro, Pourashraf, Cates and Levin. This is an openaccess article distributed under the terms of the Creative Commons Attribution License (CC BY). The use, distribution or reproduction in other forums is permitted, provided the original author(s) and the copyright owner(s) are credited and that the original publication in this journal is cited, in accordance with accepted academic practice. No use, distribution or reproduction is permitted which does not comply with these terms. 